had received therapy were interviewed face to face in Persian using a semi-structured interview guide. Interviews were audio taped, then transcribed in full, translated into English by the investigator, and analysed using MAXqda software.

Results The value men accorded to early detection of prostate cancer was found to be conditional upon their beliefs of prostate illness and their experiences about cure. There was a lack of information about the early detection process. The men felt that medical intervention was focused on the biological aspects, ignoring the needs of the psychosocial concerns. The men were not expecting to have symptoms because of prostate treatment; this influenced their subsequent decision-making.

Conclusion Given men's perceptions and experiences of the illness, screening of prostate cancer seems to have wider implications. The findings suggest that early detection of the disease in Iran may need a screening model that incorporates both biomedical and psychosocial aspects.

\section{P2-300 EFFECTS OF ANTIHYPERTENSIVE DRUG TREATMENTS ON BONE TURNOVER IN ELDERLY MEN: A CROSS-SECTIONAL ANALYSIS OF THE FUJIWARA-KYO OSTEOPOROSIS RISK IN MEN (FORMEN) STUDY}

doi:10.1136/jech.2011.142976k.33

\begin{abstract}
${ }^{1} \mathrm{~J}$ Tamaki, ${ }^{*}{ }^{1} \mathrm{M}$ Iki, ${ }^{1} Y$ Fujita, ${ }^{1} \mathrm{~K}$ Kouda, ${ }^{1} \mathrm{~A}$ Yura, ${ }^{1} \mathrm{E}$ Kadowaki, ${ }^{2} Y$ Sato, ${ }^{3} \mathrm{~J}-\mathrm{S}$ Moon, ${ }^{4} \mathrm{~K}$ Tomioka, ${ }^{4} \mathrm{~N}$ Okamoto, ${ }^{4} \mathrm{~N}$ Kurumatani. ${ }^{1}$ Department of Public Health, Kinki University School of Medicine, Osakasayama, Osaka, Japan; ${ }^{2}$ Department of Human Life, Jin-ai University, Echizen, Fukui, Japan; ${ }^{3}$ Faculty of Human Sciences, Taisei Gakuin University, Sakai, Osaka, Japan; ${ }^{4}$ Department of Community Health and Epidemiology, Nara Medical University School of Medicine, Kashihara, Nara, Japan
\end{abstract}

Introduction Previous studies have found that some antihypertensive drugs may lower fracture risk. However, the impact of antihypertensive drugs on bone metabolism is not entirely clear. We examined how antihypertensive drugs influenced bone status and bone turnover markers.

Methods We analysed 1632 Japanese men aged $\geq 65$ years from a baseline survey (the FORMEN Study) conducted during 2007-2008 as a part of the Fujiwara-kyo study, a larger prospective cohort study. Associations between antihypertensive drugs (ACE, $\beta$-blocker, and thiazide diuretic treatments) and bone metabolism (bone mineral density [BMD] at the lumbar spine [LS] and total hip, serum osteocalcin $[\mathrm{OC}]$, and serum tartrate resistant acid phosphatase isoenzyme $5 b$ [TRACP5b]) were investigated cross-sectionally.

Results Proportions of hypertension and subjects taking antihypertensive drugs were $76.0 \%(n=1240)$ and $42.4 \% \quad(n=692)$, respectively, in the subjects (mean age; 73.0 \pm 5.1 years). The numbers of subjects prescribed ACE, $\beta$-blockers, and thiazide diuretics were 62,41 , and 12 , respectively. Neither BMD nor bone turnover markers varied significantly between those with ACE prescription and those without. We observed significantly higher LS $B M D$ values and significantly lower OC and TRACP5b values in subjects taking $\beta$-blockers than in non-users, and the differences in both marker values remained significant after adjusting for confounders (OC: 4.08 [0.18] vs 4.91 [0.03] ng/ml, $\mathrm{p}=0.039$; TRACP5b: 151.23 [0.18] vs 209.98 [0.03] $\mathrm{mU} / \mathrm{dl}, \quad \mathrm{p}=0.009)$. TRACP5b values in subjects with thiazide diuretics also remained significant lower after adjusting for confounders (139.98 [0.33] vs 208.91 [0.03] mU/dl, $\mathrm{p}=0.007$ ).

Conclusion Bone turnover could be suppressed by $\beta$-blocker and thiazide diuretic treatment. Future investigations should be conducted longitudinally.

\section{P2-301 HIGH PREVALENCE OF SEDENTARINESS AMONG BRAZILIAN ADOLESCENTS LIVING WITH HIV/AIDS}

doi:10.1136/jech.2011.142976k.34

${ }^{1}$ L F Tanaka, ${ }^{1}$ M D R D de Oliveira Latorre, ${ }^{1}$ A M Silva, ${ }^{1}$ T C R Oliveira, ${ }^{1}$ E C Mendes, ${ }^{2} \mathrm{H}$ H de Souza Marques. 'School of Public Health-University of São Paulo, São Paulo, São Paulo, Brazil; ${ }^{2}$ School of Medicine, Children's Institute, University of São Paulo, São Paulo, São Paulo, Brazil

Introduction Several studies have shown high prevalences of sedentariness among adolescents, however, studies assessing sedentariness of adolescents with HIV are scarce. The aim of this study is to assess the prevalence of sedentariness in this population and its associated factors.

Methods 91 patients aged 10-19years responded to the questionnaire on physical activity validated for Brazilian adolescents. The questionnaire is comprised of 17 questions (15 on sports and two on transportation physical activity). The cut-off point for sedentariness was $300 \mathrm{~min} /$ week.

Results Mean age at interview was 15.1 years ( $\mathrm{SD}=2.6$ years). A greater proportion of girls was sedentary $(80 \% \times 61 \%, \mathrm{p}<0.05)$. All other variables tested were not associated with sedentariness: ethnicity (white-65\%; non-white- $62 \%, \mathrm{p}=0.286$ ); living with family (yes-70\%; no-89\%, $\mathrm{p}=0.220$ ); altered waist circumference (yes-70\%; no- $72 \% p=0.881$ ) and overweight (yes- $0 \%$; no- $8 \%, p=0.081$ ). No differences between means of biochemical parameters were found when comparing active and sedentary adolescents: viral load $(15995 \times 15922$ copies, $\mathrm{p}=0.995) ; \mathrm{CD} 4(485 \times 441$ cells, $\mathrm{p}=0.540)$; total cholesterol $(156 \times 162 \mathrm{mg} / \mathrm{dl}, \quad \mathrm{p}=0.523) ; \quad$ HDL-cholesterol $(39 \times 37 \mathrm{mg} / \mathrm{dl}, \quad \mathrm{p}=0.373)$ and LDL-cholesterol $(94 \times 95 \mathrm{mg} / \mathrm{dl}$, $\mathrm{p}=0.874)$. $1 / 3$ of adolescents spent no time practicing physical activity. Among those who reported practicing it, the sports most cited were: football (44.4\%), volleyball $(14.4 \%)$ and cycling $(7.8 \%)$. Mean time spent practicing sports was $198.9 \mathrm{~min} /$ week $(\mathrm{SD}=271.1$ minutes) and mean time spent walking/cycling to school was $74.1 \mathrm{~min} /$ week $(\mathrm{SD}=104.2)$.

Conclusion A high prevalence of sedentariness was found in this population. Sedentary behaviour may have a negative impact on adolescents' health.

\section{P2-302 NOCTURNAL INTERMITTENT HYPOXIA AND CARDIOVASCULAR RISK FACTORS IN COMMUNITY. DWELLING JAPANESE: THE CIRCULATORY RISK IN COMMUNITIES STUDY (CIRCS)}

doi:10.1136/jech.2011.142976k.35

${ }^{1} \mathrm{~T}$ Tanigawa, ${ }^{* 2,4} \mathrm{I}$ Muraki, ${ }^{3} \mathrm{~K}$ Yamagishi, ${ }^{1} \mathrm{~S}$ Sakurai, ${ }^{2} \mathrm{~T}$ Ohira, ${ }^{2} \mathrm{H}$ Imano, ${ }^{4} \mathrm{~A}$ Kitamura, ${ }^{4} \mathrm{M}$ Kiyama, ${ }^{5} \mathrm{~S}$ Sato, ${ }^{2} \mathrm{H}$ Iso. ${ }^{1}$ Ehime University Graduate School of Medicine, Toon, Ehime-ken, Japan; ${ }^{2}$ Osaka University Graduate School of Medicine, Suita, Osaka-fu, Japan; ${ }^{3}$ University of Tsukuba, Tsukuba, Ibaraki-ken, Japan; ${ }^{4}$ Osaka Medical Center for Health Science and Promotion, Osaka, Osaka-fu, Japan; ${ }^{5}$ Chiba Prefectural Institute of Public Health, Chiba, Chiba-ken, Japan

Introduction To investigate whether nocturnal intermittent hypoxia (NIH), a surrogate marker for sleep apnoea, is associated with cardiovascular risk factors, we conducted epidemiological studies.

Methods The CIRCS is a prospective cohort study across Japan since 1963 to examine risk factors for cardiovascular disease. Subjects aged 40-69 years were recruited to the sleep study in three communities. $\mathrm{NIH}$ was estimated by hourly occurrences of oxygen desaturation of $\geq 3 \%$ ( $3 \%$ oxygen desaturation index $[3 \%$ ODI]) by a pulse-oximeter during a night's sleep in the participant's own home. We defined no, mild and moderate-to-severe NIH by $3 \%$ ODI as $<5,5$ to $<15$ and $\geq 15$ events/h, respectively. We conducted cross sectional studies on 